\title{
28 Research Soure \\ Identification of key gene in colorectal cancer using bioinformatics analysis.
}

\section{Rongrong Xiao}

The Affiliated Hospital of Nanjing medical University https://orcid.org/0000-0002-6406-2046

\section{Ping Wang}

The affiliated Cancer Hospital of Nanjing medical university

\section{Tian Xia}

The affiliated Cancer Hospital of Nanjing medical university

\section{Chun-Yi Li}

The affiliated Cancer hospital of Nanjing medical university

\section{Ting Jiang}

The affiliated Cancer Hospital of Nanjing medical university

\section{Xin-en Huang ( $\nabla$ huangxinen006@163.com )}

The affiliated Cancer Hospital of Nanjing medical university https://orcid.org/0000-0002-9419-9166

\section{Research article}

Keywords: colon cancer, gene, TCGA, Oncomine, bioinformatics analysis

Posted Date: October 5th, 2020

DOl: https://doi.org/10.21203/rs.3.rs-63457/v1

License: (c) (1) This work is licensed under a Creative Commons Attribution 4.0 International License. Read Full License 


\section{Abstract \\ Background}

Tumor microenvironment plays important roles in the development of cancer. The aim of our study was to examine the expression of genes in colorectal cancer and also to evaluate the association value between expression level of these genes and clinical features.

\section{Methods}

We combined The Cancer Genome Atlas (TCGA) datasets to identify differentially expressed genes in colon cancer. Using these differentially expressed genes, we constructed protein-protein interaction network and conducted functional enrichment analysis. Genes with degree beyond 10 in the PPI network were regarded as hub genes. Then, we verified of the expression of molecules in Oncomine datasets and conducted Kaplan-Meier curve and log-rank test and functional enrichment analysis on these hub genes. Finally, we analyzed the relationship clinicopathological features analysis with the key gene.

\section{Results}

There were 719 differentially expressed genes identified to be associated with colon cancer microenvironment. We screened out 10 hub genes by construction of PPI network. The functions of these hub genes were enriched in cytokine-cytokine receptor interaction, alcoholism and systemic lupus erythematosus, which provided further insight into the roles of these genes in the tumor microenvironment. GNG4, with the highest degrees in the PPI network, were highly exprepressed in metastasis $(P=9.5-05), N 1(P=0.0025)$ and N2(,0.037). It was a relationship with stage. It was significantly different between with stage I and IV, II and III, II and IV,III and IV (P=0.0015,0.029,3.9-

$05,0.00074,0.01$,respectively)

\section{Conclusions}

We identified GNG4 can be regarded as a prognostic biomarker in colon cancer.

\section{Backgroud}

Colorectal cancer (CRC) is the fourth most common cancer in the world ${ }^{[1]}$. Each year 1.5 million people worldwide are diagnosed with CRC. In America around 60,000 deaths due to CRC happens ${ }^{[1]}$. Approximately $30 \%-50 \%$ of patients will suffer recurrence despite achieving remission with treatment ${ }^{[1]}$. Approximately $20-25 \%$ of patients with colorectal cancer (CRC) have distant organ metastasis at the time of initial diagnosis ${ }^{[2]}$ Accumulating evidence also has demonstrated that multiple genes and cellular 
pathways participate in the occurrence and development of CRC using bioinformatics analysis ${ }^{[3]}$.These findings have inspired the new discovery of molecularly targeted therapies as a novel approach for CRC treatment. Understanding the molecular mechanism involved in proliferation, apoptosis, and invasion of $\mathrm{CRC}$ are therefore imperative. Functional enrichment analysis revealed potential biological functions and signal pathways of mRNA signature associated with cancer, which enhances our understanding to molecular mechanisms of model in CRC. In this study, we aimed to explore the difference in the mRNA expression profiles of CRC to identify potential gene biomarkers using TCGA data. We established one gene-GNG4 prognostic model that based on our RNA sequencing analysis. The model was validated in Oncomine. We found GNG4 was associated with clinical characteristics. As a whole, GNG4 might be helpful in guiding the prognostic status of patients.

\section{Methods}

\section{Data collection}

The mRNA sequence data and clinical characteristics were downloaded from the publicly available TCGA database (https://cancergenome.nih.gov/). The data processing was in accordance with the publication guidelines provided by TCGA(http://cancergenome.nih.gov/publications/publicationguidelines).All data were acquired from the TCGA database. Herein, ethical committee assessment is not required for the study.

Identification of differentially expressed genes

The package 'edgeR' version3.6.2(http://www.bioconductor.org/package/release/bioc/

heml/edgeR.html) in R version 3.6.1. ${ }^{[4]}$ was used to identify differential gene between CRC and normal tissues. The miRNA that could not be determined in all samples were excluded. A llog2 fold change $(\log 2 \mathrm{Fc}) \mid$ of $>2, \log 2 \mathrm{Fc}<-2$ and an adjusted $\mathrm{P}$-value of $<0.05$ were considered as the cut-off criteria. The expression levels of the mRNAs were log2 transformed for further analysis.

Functional enrichment analysis

Using ggplot2 package ${ }^{[5]}$, we conducted gene ontology (GO) and Kyoto Encyclopedia of Genes and Genomes (KEGG) pathway analysis. $G 0$ terms of biological process (BP), cellular components and molecular functions, and KEGG pathways with FDR-adjusted P-value $<0.05$ were considered to be significant.

Construction of protein-protein interaction network

Using STRING database ${ }^{[6]}$ (http://string-db.org/, version 11.0), we construct the PPI network of gene. Gene-gene interactions with integrated scores bigger than 0.90 were selected and visualized using Cytoscape (version 3.8.0) ${ }^{[7]}$. The degree of genes indicated the number of edges (interactions) that linked 
to a given node (gene). We calculated the degree of each gene and selected the hub genes who are top 10 in network string interactions.

Verification of the expression of molecules

The differential molecules were validated using the colon cancer microarray data set in the Oncomine Platform(https://www.oncomine.org/resource/main.html.) ${ }^{[8]}$.The Oncomine Platform

(http://www.oncomine.org) has identified the genes, pathways, and networks deregulated across 18,000 cancer gene expression microarrays, spanning the majority of cancer types and subtypes ${ }^{[8]}$. Thus, we adopted the Oncomine database to further validate the expression of differentially expressed genes. The differential expression analysis, coexpression analysis and meta-analysis was directly performed using Oncomine online analysis tools.

Kaplan-Meier (K-M) curve and clinicopathological features analysis

Kaplan-Meier survival analysis ${ }^{[9]}$ were then performed to assess the difference in OS between low $<$ median of expression levels) and high level (>= median of expression levels) groups. The survival analyses were accomplished by using the $\mathrm{R}$ package "survival" ${ }^{[10]}$. We further analyzed the relationship between mRNAs and clinicopathological features by using "ggpubr" packages ${ }^{[11]}$.

\section{Results}

Clinical information of CRC patients from the TCGA

Database was available in 539 cases. The clinical characteristics including age, gender, tumor stage ,and lymph node metastasis of CRC patients are presented in Table 1. The median age of the cohort was 68 years(range: $31-90$ years). 
Table 1

The clinicopathological characteristics of CRC patients.

\begin{tabular}{|llll|}
\hline \multirow{2}{*}{ Age } & $<=65$ & 223 & $41.37 \%$ \\
\cline { 2 - 4 } gender & $>65$ & 316 & $58.63 \%$ \\
\cline { 2 - 4 } Tumor Stage & Female & 256 & $47.50 \%$ \\
\cline { 2 - 4 } & Male & 283 & $52.50 \%$ \\
\cline { 2 - 4 } & II & 91 & $16.88 \%$ \\
\cline { 2 - 4 } & III & 156 & $36.55 \%$ \\
\cline { 2 - 4 } Lymph node & IV & 76 & $14.10 \%$ \\
\cline { 2 - 4 } & N1 & 305 & $56.59 \%$ \\
\cline { 2 - 4 } & N2 & 125 & $23.19 \%$ \\
\hline M & M0 & 394 & $73.10 \%$ \\
\cline { 2 - 4 } & M1 & 76 & $14.10 \%$ \\
\hline N = 539 & & & \\
\hline Missing values wasn't presented in Table. \\
\hline
\end{tabular}

Identification of differentially expressed genes

A total of 514 mRNA samples were enrolled in our study, including 473 colon carcinoma tissues and 41 matched normal tissues. To pick out the differentially expressed genes with normal issues and CRC, we conducted differentially expression analysis. We filtered low expression genes. To date,719 mRNAs were found to be significantly upregulated and downregulated in CRC. For differentially expressed genes, we identified 375 upregulated genes and 344 downregulated genes. The heatmap showed 50 samples by the differentially expressed genes (Fig. 1). The volcano plot showed the relationship between FDR(false discovery rate) and Fold Change(FC) in all differentially expressed genes(Fig. 2).It showed normal and cancer group could be clearly distinguished by the differentially expressed genes.

Functional enrichment analysis of differentially expressed genes

To further investigate the biological functions of these differentially expressed genes, we conducted functional enrichment analysis. The BP(biological process )terms, CC(cellular components)terms and MF(molecular functions) terms were exhibited in Fig. 3A and Table 2. Proteolysis,protein heterodimerization activity and extracellular exosome were the main enriched GO(gene ontology) terms. 
KEGG(Kyoto Encyclopedia of Genes and Genomes) pathways were displayed in Fig. 3B. The main enriched pathways was cytokine-cytokine receptor interaction, alcoholism and systemic lupus erythematosus.

Protein-protein interaction (PPI) network and identification of hub genes

The PPI network of differentially expressed genes contained 715 nodes and 1186 edges. We displayed the sub-network with most nodes and edges in Fig. 4. The average node degree is 3.32. Expected number of edges is 532.PPI enrichment p-value $<1.0^{-16}$. According to the PPI network, my network has significantly more interactions than expected what means functional enrichments in my network. The degree of these genes was shown in Table 3. Top 10 genes (degree $>/=25$ ) were selected as hub genes (Fig. 5),including:GNG4,PPBP,NMU,LPAR1,CXCL1,HIST1H2BJ,CXCL8,PYY,HIST1H2AD and SST.GNG4 could be the key genes in colorectal cancer using bioinformatics analysis.

GNG4 expression difference validation based on the Oncomine database

Four CRC datasets in the Oncomine database were adopted for the validation of gng4 overexpression(Skrzypczak Colorectal2 Statistics[http://www.ncbi.nlm.nih.gov/geo/query/acc.cgi? acc=GSE20916];Kaiser Colon Statistics[http://www.ncbi.nlm.nih.gov/geo/query/acc.cgi?acc=GSE 5206];Hong Colorectal Statistics [http://www.ncbi.nlm.nih.gov/geo/query/acc.cgi? acc=GSE9348];Skrzypczak Colorectal Statistics[http://www.ncbi.nlm.nih.gov/geo/query/acc.cgi? acc=GSE 20916]), which was in accordance with the results of the meta-analysis (Fig. 6A,6B,6C,6D). The P-values for these datasets were $1.13^{-10}, 8.31^{-7}, 7.09^{-9}$ and3.53 ${ }^{-5}$,respectively. However, the remaining datasets did not show a statistically significant difference in GNG4 expression. The results of GNG4 copy number in TCGA Colorectal 2 Grouped by overall survival status was showed in Fig. 7 (http://tcgadata.nci.nih.gov/tcga/). The median rank is 1219.5 after comparison of GNG4 across 4 analyses( $P=$ 4.19-7,Fig. 11) by meta-analysis. Shows that GNG4 overexpression was statistically significant. GNG4 coexpression results were shown in Fig. 8.

Analysis of clinical magnitude

The clinical magnitude of GNG4 in CRC was assessed on the basis of TCGA clinical data. A total of 539 patient samples with CRC were retrieved from a cohort of TCGA database. The association of gene expression with tumor stage, lymph node, metastasis, age, gender was analyzed. It was significantly different between with stage I and IV, II and III, II and IV,III and IV ( $P=0.0015,0.029,3.9^{-}$ ${ }^{05}, 0.00074,0.01$,respectively) (Fig. 9A). It was a relationship with stage. It was a relationship with lymph node, where the frequency of GNG4 was significantly different in patients with $\mathrm{N} 1, \mathrm{~N} 2$ than $\mathrm{NO}(P=0.0025$, 0.037)( Fig. 9B). We observed that GNG4 was highly expressed in metastasis patients $\left(P=9.5^{-05}\right.$, Fig. 9C).Taken together, GNG4 was associated with stage, metastasis and lymph node. In other words, GNG4 maybe a prognostic molecule. GNG4 expression did not show a statistically significant difference in in age and gender( $P=0.16,0.1$, respectively) (Fig. 9D,9E). There wasn't statistically different between I 
and II,I and III may due to the small number of stage I patients. There wasn't statistically different in survival curve of GNG4 expression between low level group and high group $(P=0.96641$, Fig. 10$)$.We should collect clinical patients to validate.

\section{Disccussion}

Understanding the molecular mechanism of CRC is of very importance for diagnosis and treatment. With the rapid development of information technology, the ability to collect genomic and clinical information can be used to study disease progression and improve medical treatment ${ }^{[12]}$. The causes of cancer progression are complex and diverse. Signaling pathways, covering a series of actions among multiple molecules occurring within cells, are important biological mechanisms in cell growth as well as proliferation ${ }^{[13,14,15]}$. Discovering how the pathways and the molecules therein are associated with cancer is one of most essential problems for cancer researchers in the past decades. ${ }^{[16,17]}$ Our data provide a comprehensive bioinformatics analysis of differentially expressed genes, which play crucial roles in CRC progression. Discovering gene expression patterns in oncogenes potentially can provide a reliable instrument for treatment decision and management of CRC patients. The aim of our study was to examine the expression of GNG4 gene in colorectal cancer and also to evaluate the association value between expression level of these genes and clinical features. We collected and analyzed the data on patient's gender and age, gender, lymph node involvement, distant metastasis and overall-survival. There was no statistically significant association between overall-survival and expression pattern of genes of interest. Therefore, GNG4 were not a significant prognostic factor for overall survival of patients with CRC. OS (overall survival) could be affected by different treatment methods. We should explore more to collect clinical patients to analysis association between GNG4 with OS or PFS(progress free survival) by stratum in keeping with the predetermined therapy plan. However, we found GNG4 was statistically significant association between GNG4 expression and lymph node involvement, distant metastasis. It reveals that GNG4 could be one prognostic marker to remind whether these patients with high-level expression are easier to progress. And it could be one target of treatment to block the disease progress. Admittedly, our study has some limitations because it was based on the bioinformatics analysis only. The roles of these hub genes deserve further in vitro and in vivo studies. More research with larger samples on this topic needs to be undertaken.

\section{Conclusions}

We found GNG4 was associated with clinical characteristics. We identified GNG4 can be regarded as a prognostic biomarker in colon cancer. GNG4 could be one prognostic marker to remind whether these patients with high-level expression are easier to progress.

\section{Abbreviations}

CRC colon cancer 
OS

overall survival

PFS

progress free survival

GO

gene ontology

KEGG

Kyoto Encyclopedia of Genes and Genomes

$\mathrm{BP}$

biological process

CC

cellular components

MF

molecular functions

FDR

false discovery rate

FC

Fold Change

\section{Declarations}

\section{Ethics approval and consent to participate}

The data and clinical characteristics were downloaded from the publicly available TCGA database (https://cancergenome.nih.gov/) and validated in oncomine(https://www.oncomine.org/resource/main.html.).Herein, ethical committee assessment and consent to participate is not required for the study.

\section{Consent for publication}

this manuscript is approved by all authors for publication.

\section{Competing interest}

No conflict of interest exits in the submission of this manuscript

\section{Funding}

This paper was supported by the Foundation of the affiliated Cancer Hospital of Nanjing medical university [ZM201901]. The funding body did not play any roles in the design of the study, collection, analysis, and interpretation of data, and in writing the manuscript.

\section{Authors' contributions}


All authors have read and approved the manuscript. All authors have contributed significantly and in keeping with the latest guidelines of the International Committee of Medical Journal Editors, each author's contribution to the paper has been described in the following:

RX: contributed to the conception of the study and manuscript preparation,performed the data analyses and wrote the manuscript;

PW: helped perform the analysis with the data analyses and constructive discussions.

TX: helped perform the analysis with constructive discussions.

CL: helped perform the analysis with constructive discussions.

$\mathrm{TJ}$ : helped perform the analysis with constructive discussions.

$\mathrm{XH}:$ Revised the manuscript and helped perform the analysis with constructive discussions.

\section{Acknowledgements}

This study is supported by the Affiliated Cancer Hospital of Nanjing Medical University. It was also supported by the public databases of The Cancer Genome Atlas (TCGA) and Oncomine Platform.

\section{Availability of data and materials}

All data generated or analysed during this study are included in this published article.

\section{References}

1. Tavakoli AK, Mahjoubi B, Mirzaei R, et al. AKAP4, SPAG9 and NY-ESO-1 in Iranian Colorectal Cancer Patients as Probable Diagnostic and Prognostic Biomarkers[J]. Asian Pacific Journal of Cancer Prevention Apjcp. 2018;19(2):463-9.

2. Kim IY, Kim BR, Kim HS, Kim YW. Differences in clinical features between laparoscopy and open resectionfor primary tumor in patients with stage IV colorectal cancer[J]. Onco Targets Ther. 2015;8:3441-8.

3. Zhang T, Guo J, Gu J, Wang Z, Wang G, Li H, Wang J. Identifying the key genes and microRNAs in colorectal cancer liver metastasis by bioinformatics analysis and in vitro experiments[J]. Oncol Rep eports. 2019;41(1):279-91.

4. Mark D, Robinson DJ, McCarthy GK. Smyth:edgeR: a Bioconductor package for differential expression analysis of digital gene expression data[J]. Bioinformatics. 2010;26(1):139-40.

5. Yu G, Wang LG, Han Y, He QY. clusterProfiler: An R package for comparing biological themes among gene clusters[J]. OMICS. 2012;16(5):284-87.

6. Szklarczyk D, Gable AL, Lyon D, et al. STRING v11: protein-protein association networks with increased coverage, supporting functional discovery in genome-wide experimental datasets[J]. 
Nucleic Acids Res. 2019;47(D1):D607-13.

7. Otasek D, Morris JH, Bouças J, et al. Cytoscape Automation: empowering workflow-based network analysis[J]. Genome Biol. 2019;20(1):185-.

8. Rhodes DR, Kalyana-Sundaram S, Mahavisno V, et al. Oncomine 3.0: genes, pathways, and networks in a collection of 18,000 cancer gene expression profiles[J]. Neoplasia. 2007;9(2):166-80.

9. Goel MK, Khanna P, Kishore J. Understanding survival analysis: Kaplan-Meier estimate[J]. Int J Ayurveda Res. 2010;1(4):274-8.

10. Therneau TM, Lumley T. Package 'survival'[J]. Survival analysis Published on CRAN. 2014;2:3.

11. Kassambara A. ggpubr:"ggplot2” based publication ready plots. R package version 0.1. 6[J]. 2017.

12. Urits I, Gress K, Charipova K, et al. Diagnosis, Treatment, and Management of Dejerine-Roussy Syndrome: a Comprehensive Review[J]. Curr Pain Headache Rep. 2020;24(9):1-9.

13. Roux PP, Topisirovic I. Signaling pathways involved in the regulation of mRNA translation[J]. Molecular cellular biology. 2018;38(12):e00070-18.

14. Menter DG, Davis JS, Broom BM, et al. Back to the colorectal cancer consensus molecular subtype future[J]. Curr Gastroenterol Rep. 2019;21(2):5.

15. Warns J, Marwarha G, Freking N, et al. 27-hydroxycholesterol decreases cell proliferation in colon cancer cell lines[J]. Biochimie. 2018;153:171-80.

16. Sun Y, Mironova V, Chen Y, et al. Molecular Pathway Analysis Indicates a Distinct Metabolic Phenotype in Women With Right-Sided Colon Cancer[J]. Translational oncology. 2020;13(1):42-56.

17. De Palma FDE, D'argenio V, Pol J, et al. The molecular hallmarks of the serrated pathway in colorectal cancer[J]. Cancers. 2019;11(7):1017.

\section{Tables}

Due to technical limitations, table 2 and 3 is only available as a download in the Supplemental Files section.

\section{Figures}




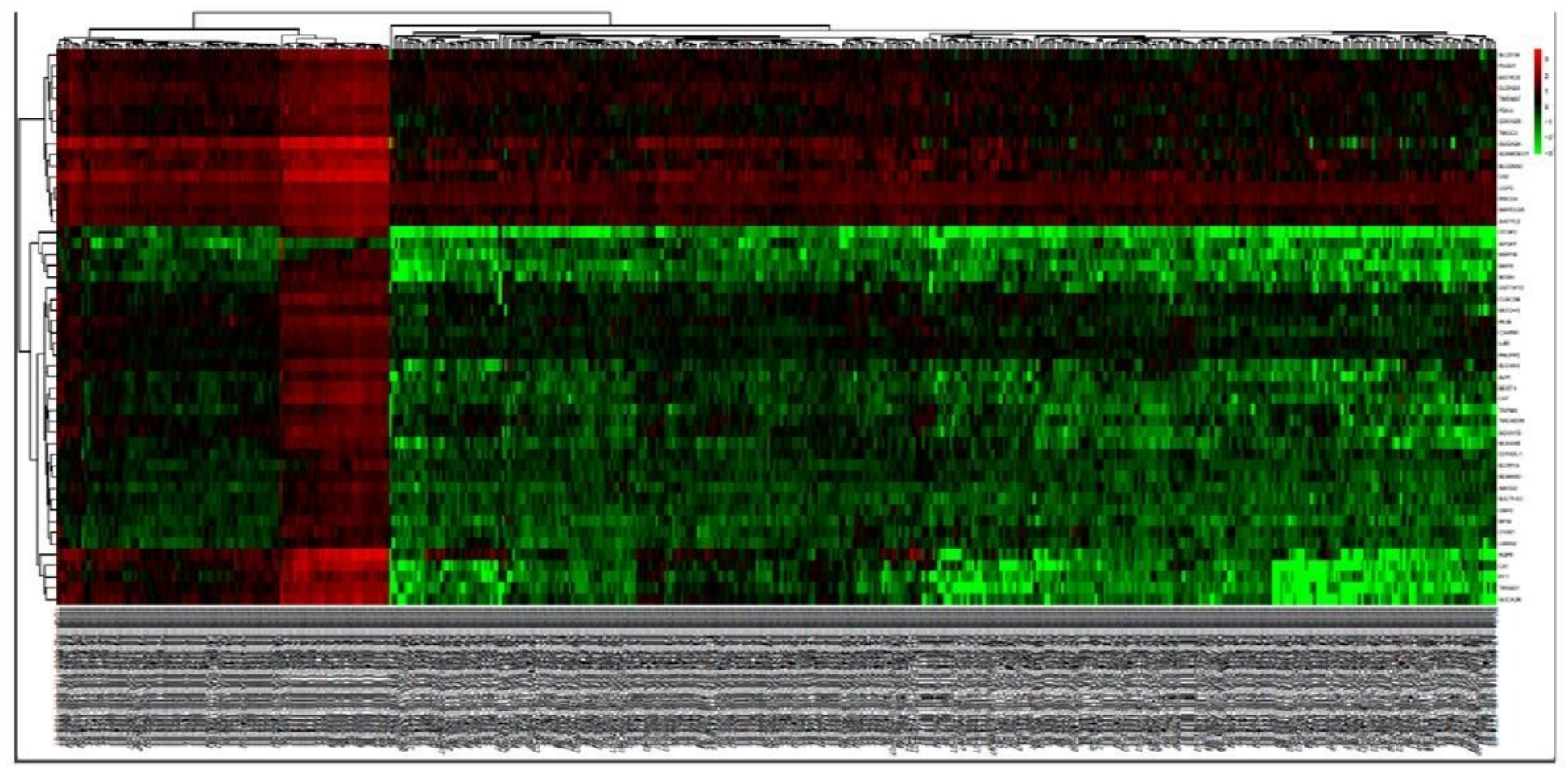

\section{Figure 1}

Heatmap of differentially expressed genes. 


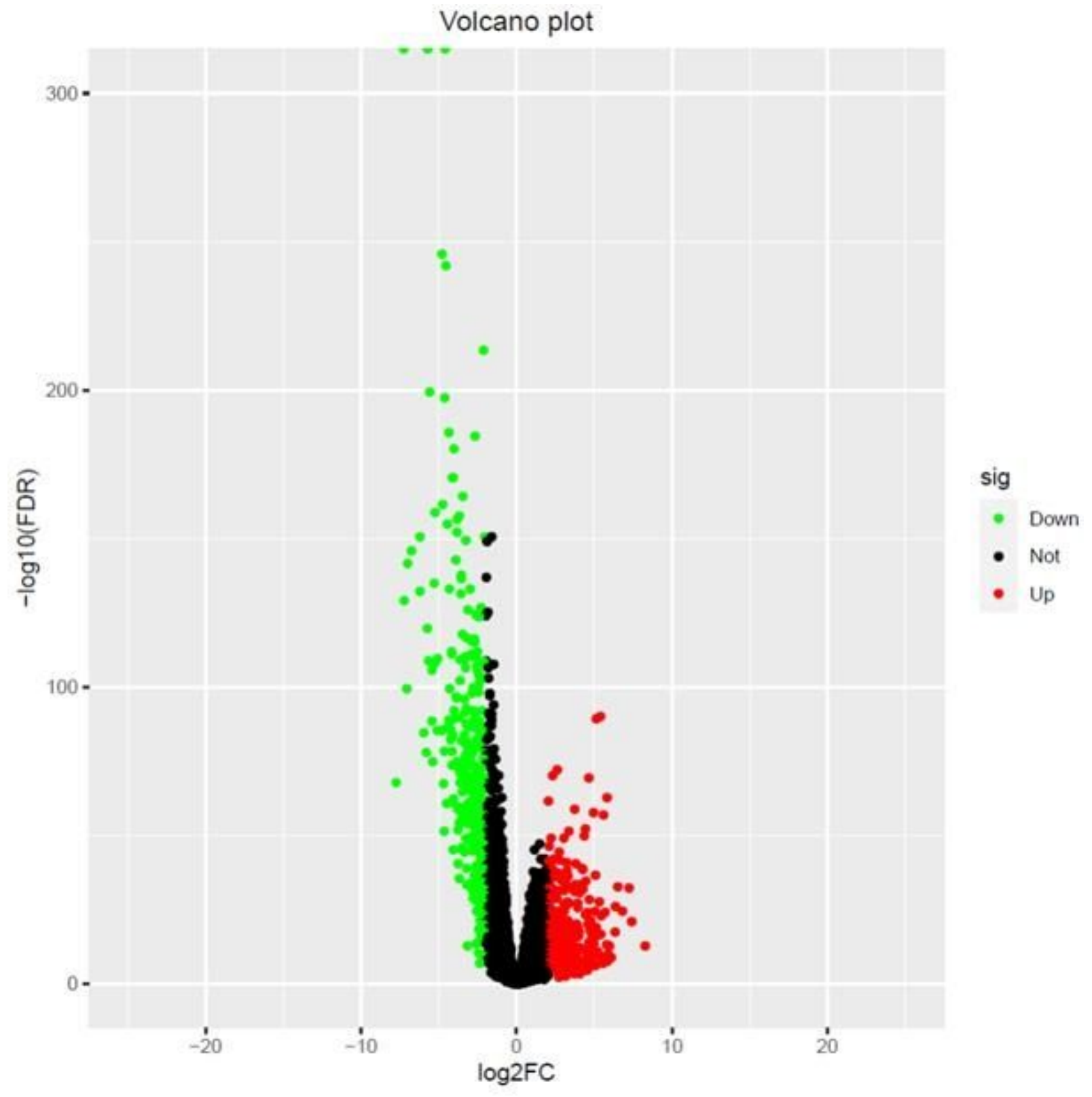

Figure 2

The volcano plot: the relationship between FDR and FC in all differentially expressed genes. 

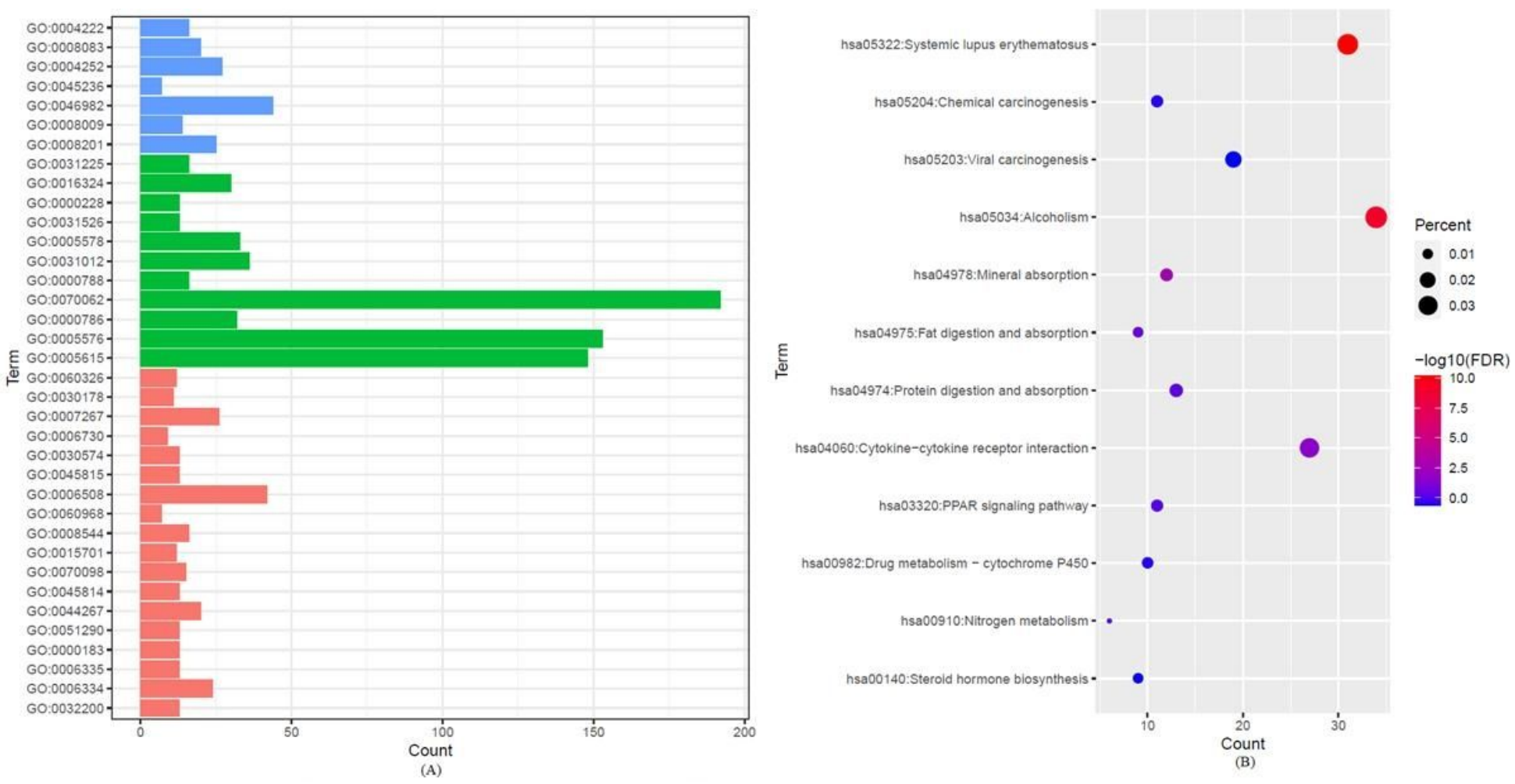

Figure 3

Functional enrichment analysis of differentially expressed genes. (A)GO terms of biological process (BP), cellular components and molecular functions. (B)Kyoto Encyclopedia of genes and genomes pathways.

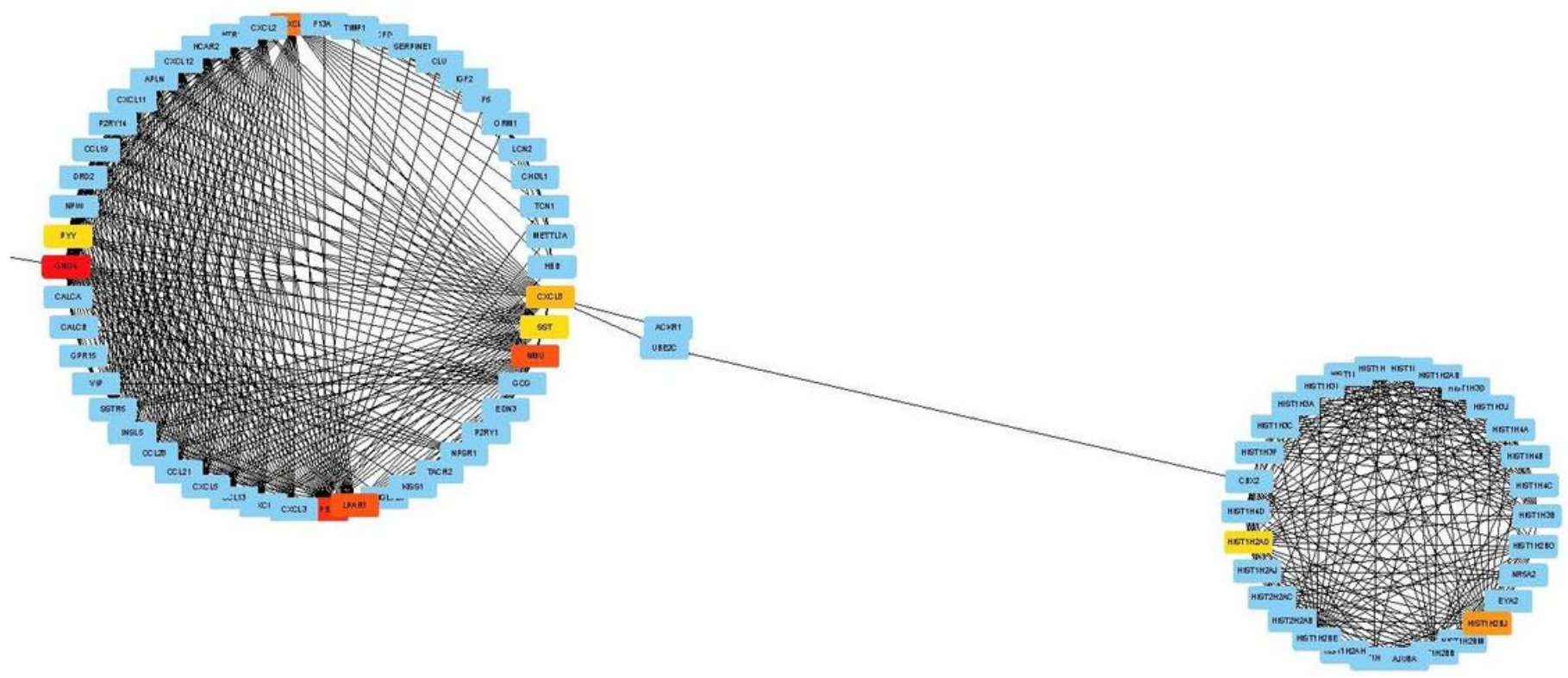

Figure 4

The PPI network of differentially expressed genes. 

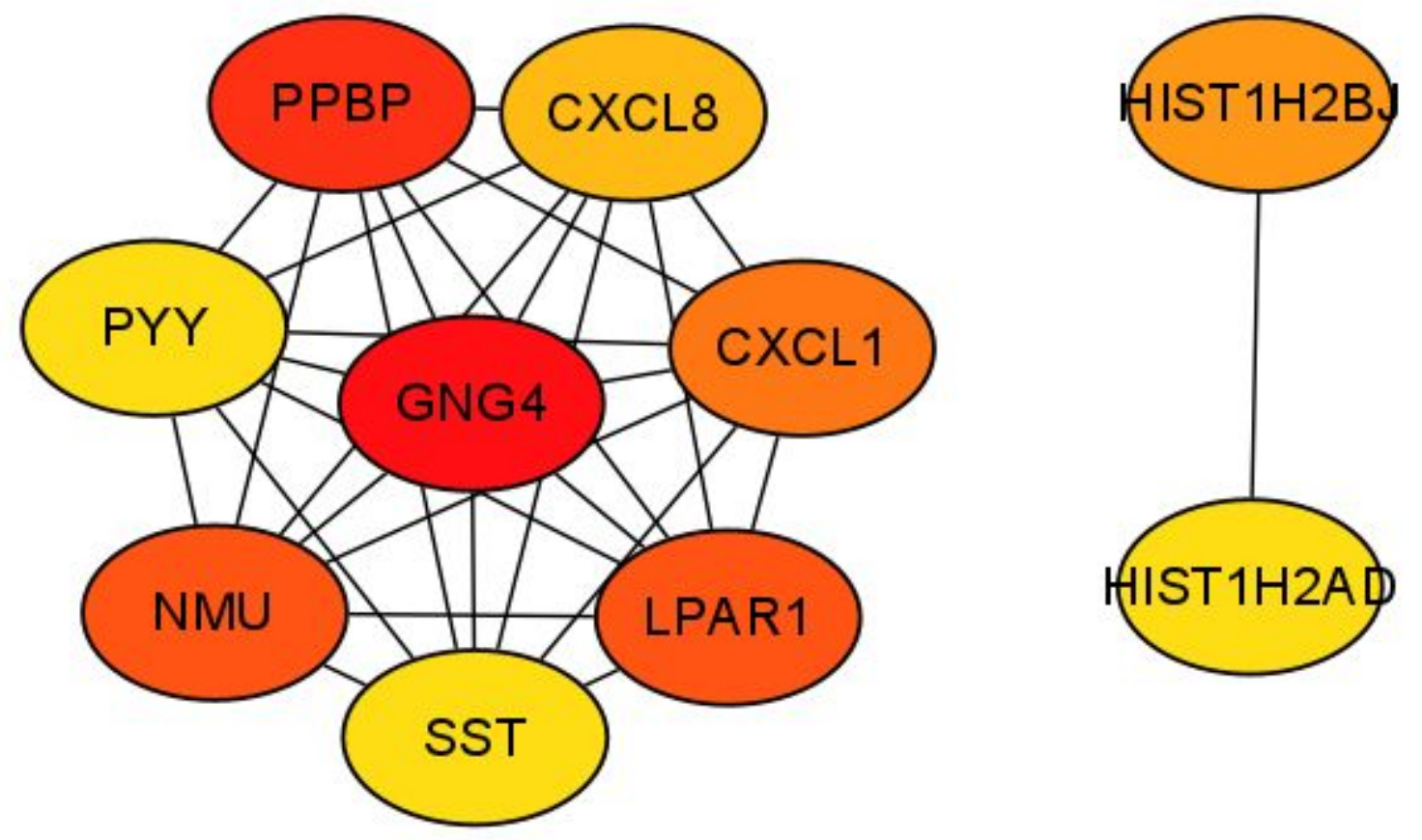

Figure 5

Hub genes. 
$\omega$ GNO4 Expression in Skrzypczak Colorectal 2

Skrzypczak Colorectal 2 Statistics

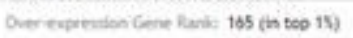
Depontier 1555657 at $\cdot$ Pivalue: 1,13E-10

1.Tes: 17.502

foldochanes: 9.730

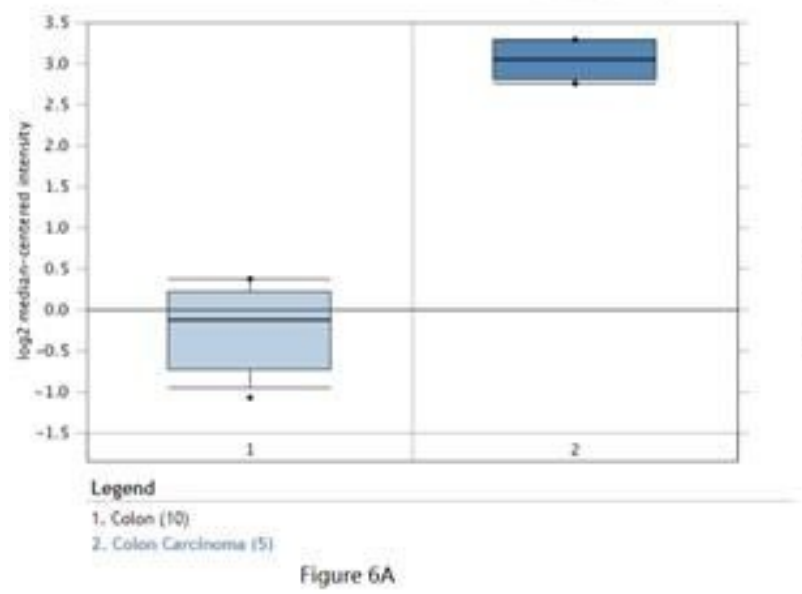

WN GNG4 Expression in Hone Colorectal

Hong Colorectal Statistics

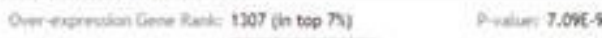

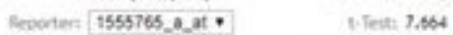

Fold Ohaser: 8.838

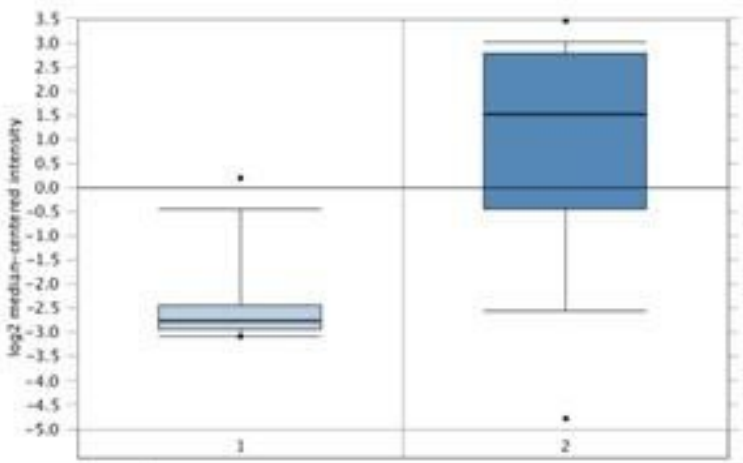

Legend

1. Colion (12)

2. Colorectal Cancinoms gro

Figure $6 \subset$
Wy GNG4 Expression in Kaiser Colon

Colon Adenocarcinons rs. Normal

Kalser Colon Statistics

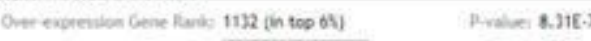

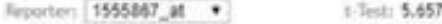

Fold Chaneer 2.39

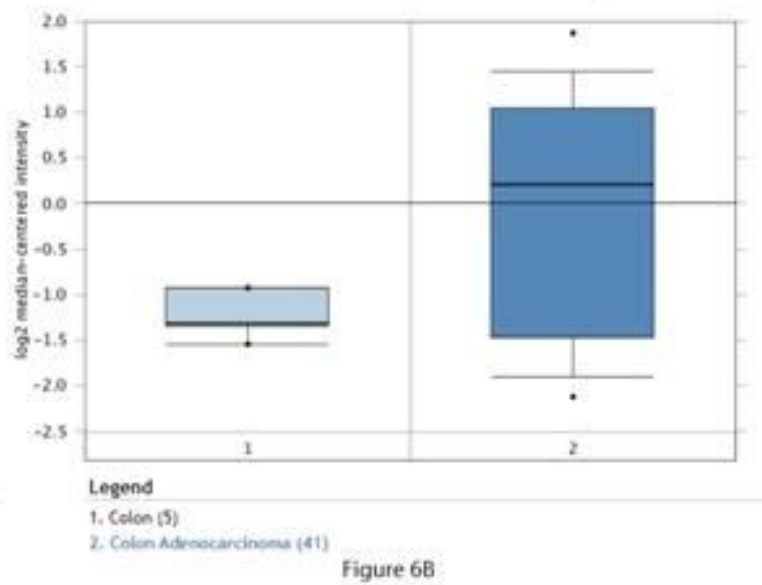

GNG4 Expression in Skrzypczak Colorectal Colorectal Cavcinoma vas. Normal

Skrrypcrak Colorectal Statistics

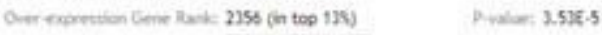
Reporters 1555765_at * tiTene 4.420

Fold Chaner: 1.964

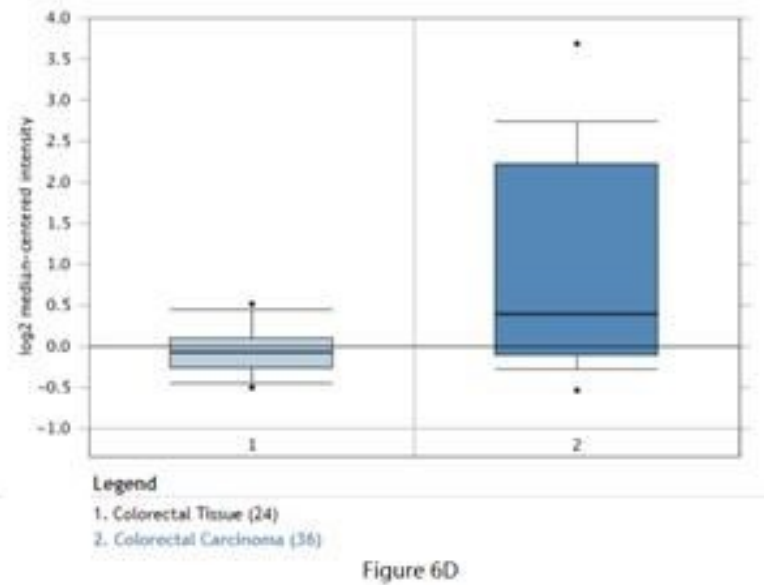

\section{Figure 6}

GNG4 expression difference validation based on the Oncomine database.<smiles></smiles>

\section{Figure 7}

The results of GNG4 copy number. 
Genes Coexpressed with GNG4 in TCGA Colorectal

Grouped by Cancer Type

( $\log 2$ median-centered ratio)

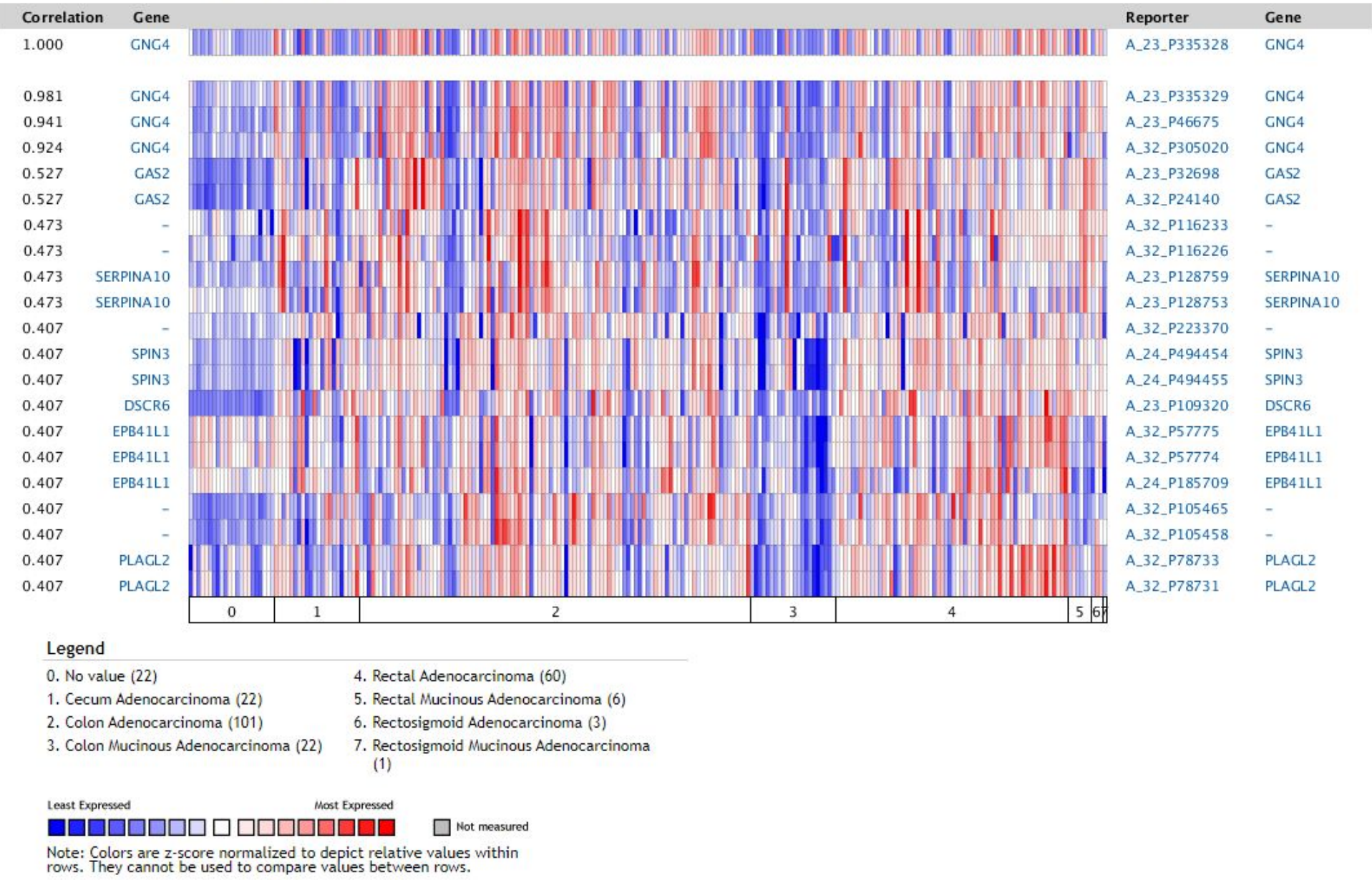

\section{Figure 8}

GNG4 co-expression results. 

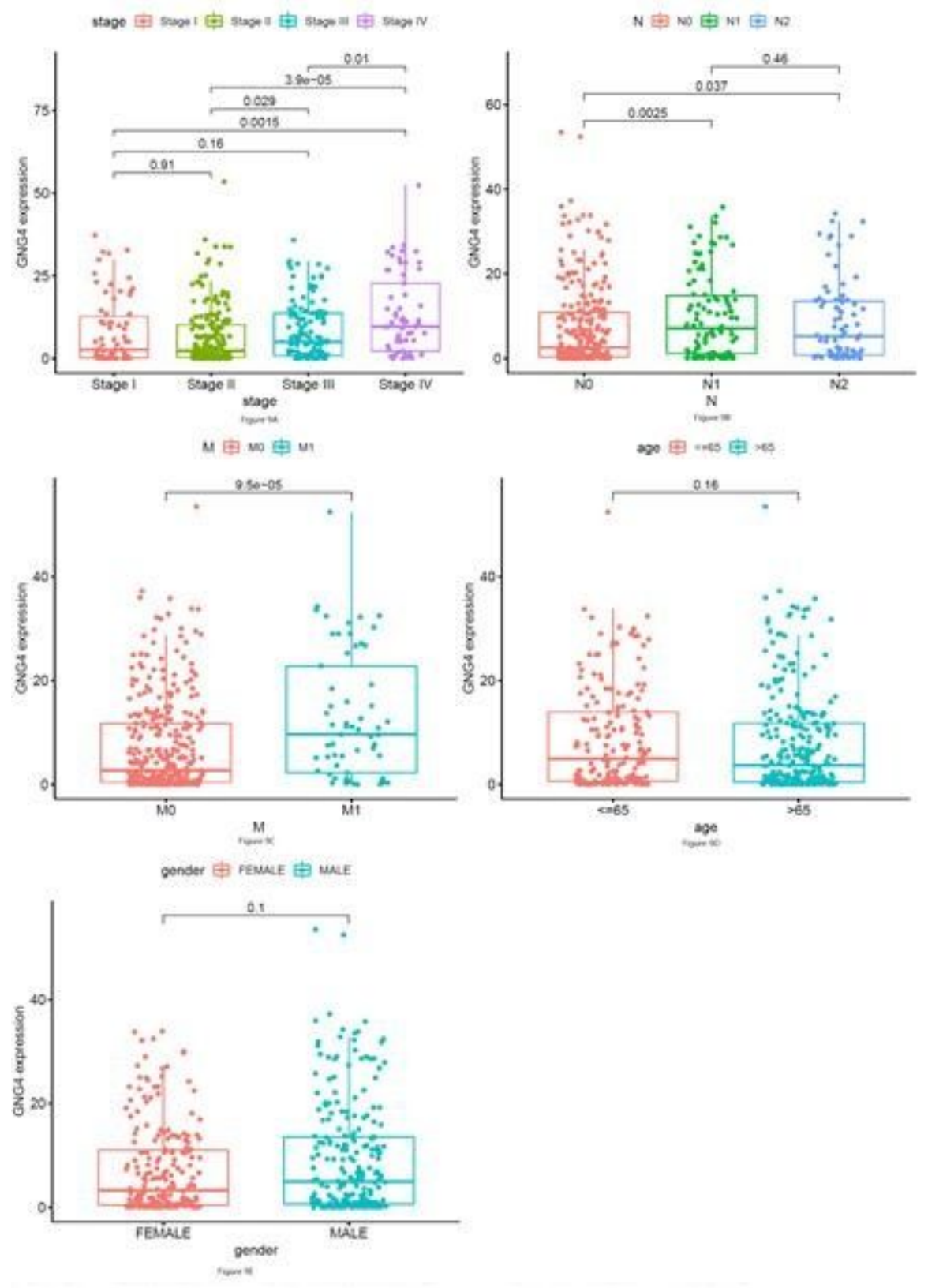

\section{Figure 9}

The clinical magnitude of GNG4 in CRC. (A) The relationship between GNG4 with stage.(B) The relationship between GNG4 with lymph node.(C) The relationship between GNG4 with metastasis.(D) The relationship between GNG4 with age.(E) The relationship between GNG4 with gender. 


\section{Surival curve of GNG4 expression ( $\mathrm{P}=0.96641)$}

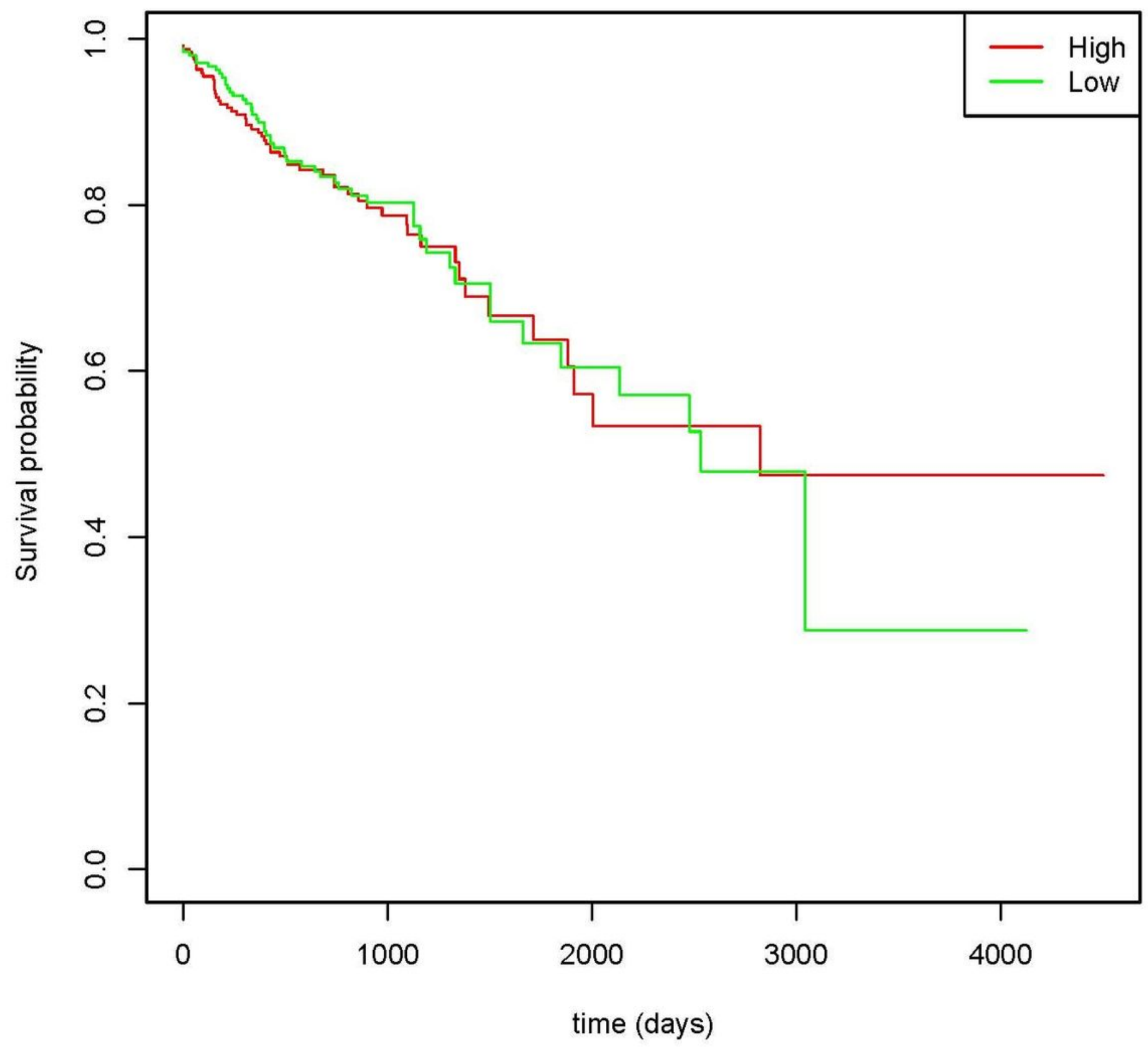

Figure 10

Surival curve of GNG4 expression. 


\section{Over-expression}

\section{Median Rank p-Value Gene \\ $1219.5 \quad 4.19 \mathrm{E}-7$ GNG4

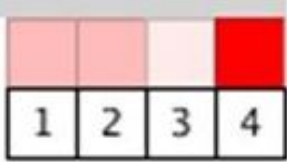

\section{Legend}

1. Colorectal Carcinoma vs. Normal Hong Colorectal, Clin Exp Metastasis, 2010

2. Colon Adenocarcinoma vs. Normal Kaiser Colon, Genome Biol, 2007
3. Colorectal Carcinoma vs. Normal

Skrzypczak Colorectal, PLoS One, 2010

4. Colon Carcinoma vs. Normal

Skrzypczak Colorectal 2, PLoS One, 2010

\section{$\begin{array}{llllllll}1 & 5 & 10 & 25 & 25 & 10 & 5 & 1\end{array}$ \\ $\square \square \square \square \square \square \square \square \square \square$ Not measured}

The rank for a gene is the median rank for that gene across each of the analyses.

The $\mathrm{p}$-Value for a gene is its $\mathrm{p}$-Value for the median-ranked analysis.

\section{Figure 11}

Comparison of GNG4 across.

\section{Supplementary Files}

This is a list of supplementary files associated with this preprint. Click to download.

- clinical.xls

- Table3.xlsx

- Table2.xlsx 